\title{
Adaptive Sustainable Organizations: System Dynamic Models of a University Balancing the Virtuous and the Vicious
}

\author{
Bhavya Patel \\ University of Auckland \\ bpat239@aucklanduni.ac.nz
}

\author{
Gabrielle Peko \\ University of Auckland \\ g.peko@auckland.ac.nz
}

\author{
David Sundaram \\ University of Auckland \\ d.sundaram@auckland.ac.nz
}

\begin{abstract}
Global business is becoming increasingly complex and is characterized by rapid and unpredictable change. This unpredictability means that organizations are being challenged at all levels. Customers, employees, partners, investors and society are all sources of uncertainty resulting in the need for organizations to be adaptive. Traditional deliberate strategies based on cycles of stability and predictability are no longer relevant for today ${ }^{1}$ business environments. Emergent strategies have been proposed by many as the answer. However, this research explores the need for organizations to interweave the deliberate with the emergent in terms of the key behavioral flows of information, learning and control for an organization to be truly adaptive. We propose a systems view of an Adaptive Sustainable Organization and we illustrate this using a research driven University as an example. Furthermore, we build system dynamic models to illustrate the vicious and virtuous cycles that could occur in such a University context.
\end{abstract}

\section{Introduction}

In a world of constantly evolving environments, it is crucial that organizations ensure their operations and practices are both adaptive and sustainable. Strategies surrounding adaptability and sustainability have become a necessity in order to remain aligned with the ever-changing environment and is therefore important to consider. This paper seeks to explore the concept of an Adaptive Sustainable Organization (ASO) and its place in today's world. To do this, firstly the various perspectives of an organization are considered separately and then evaluated in conjunction with one another to develop a holistic systems view. To illustrate how this view may apply, the operations of a tertiary education institution in the form of a university. There is investigation into what constitutes an adaptive and sustainable university and what relationship between the primary activities of researching and teaching is. In continuing the application of the systems view, a series of models are presented which demonstrate a systems thinking approach to ASOs. The models are elaborated and discussed in detail to illustrate the systems view.

\section{Perspectives on Organizations}

The construction of a systems view of an ASO requires consideration of multiple perspectives. In particular there must be focus on the Hierarchical, Functional, and Systems view of an organization. This sets the foundation for the discussion of adaptive and sustainable perspectives. This in turn will allow for a systems perspective of an ASO to be considered. The key literature to consider here is the work of Anthony [1], Scott Morton [2], Von Bertalanffy [3], and more recently Peko et al. [4]. The perspectives and views are derived from these sources of literature and the relevant frameworks are alluded to in the following sections.

\subsection{Hierarchical View}

The hierarchical view of an organization is focused on the various levels of an organization and how they integrate with each other. The core seminal literature that illustrates this view is Anthony's [1] work on organizational management which proposes a framework detailing the specific layers. Anthony's model highlights how an organization can be considered in terms of the strategic, tactical, and operational level. Viewing an organization with respect to these levels shows how it is crucial for an organization to integrate across these levels to achieve its objectives. The key dimension to consider here is that the model alludes to a systematic way of operation as the strategic level must align with other levels for the organization to operate effectively. Seminal literature such as Anthony's [1] therefore acts as a platform for the systems view to be considered. 


\subsection{Functional View}

The functional view of an organization primarily perceives an organization as a collection of departmental silos. This traditional perspective views these silos as interconnected through the flow of data [5]. A key seminal framework which illustrates the functional view and its integration is Scheer's 'Systems in an Organization' framework [6]. This framework forms a representative landscape of an integrated system, and associated sub-systems, which are used to monitor activities right through to the lowest level of the organization. They are essential for an organization at both the horizontal and vertical (functional) levels of the organization's structure.

Furthermore, Scheer's framework [6] highlights the cross functional relations and suggests that there is efficiency gain through these relations, particularly if the relevant infrastructure exists. The important fact to consider here is that a functional view is focused on deriving efficiency through integration of specialization. The logic is that functions/departments colluding for a common objective will achieve better outcomes than if the functions lack integration.

\subsection{Systems View}

A systems view is focused on the ideology that an organization operates as a system. Scott Morton [2] first conceptualized that an organization may be regarded as a complex system driven by four key elements: Strategy, Organizational Structure, Business Processes, and Information Systems. These factors are commonly known as the SOPI factors and Scott Morton proposed that it was the interrelation between these factors that operations were conducted upon. Since then, developments of the systems view has been oriented towards the cybernetics domain. One of the first theories proposing the concept of a cybernetic system was by Von Bertalanffy [3] who described the system as an adaptive and self-regulating through feedback loops. The consideration of a cybernetic system is therefore important as elements of adaptability are inherent in the concept. Peko [7] supports this idea and also ties the SOPI factors into the concept by suggesting that the factors reflect a cybernetic system.

\subsection{Adaptive View}

Scott-Morton [2] suggests that an organization can be thought of as a complex system comprised of interrelated forces that is constantly adapting to influences from both its external and internal environment. This interrelated system, that is the organization, can be thought of in terms of strategy, structure, processes, individuals and roles, and technologies. These elements, together with the influence of culture and the external socioeconomic and external technological environment, enable an organization to function and evolve. These adaptive processes and structures should be populated by adaptive individuals in composite flexible roles and the five elements together need to be supported by systems and technologies that have inherent capabilities of adapting.

Scheer [8] proposes a model which illustrates the intensity of control versus connectivity between organizational groups both internally and externally. Scheer [8] suggests that organizations with traditional, top down, hierarchical management structures have high levels of intensity of control and low connectivity. These organizations are inflexible, but succeed in stable environments and follow a deliberate approach. Conversely, organizations that follow an emergent approach are at the bleeding edge and are very reactive and flexible. Their levels of connectivity are very high while the intensity of control is very low. Scheer [8] also suggests that the best place to be is on the edge of chaos where organizations balance flexibility and stability. This equates to a balance between the deliberate and emergent approach. The edge of chaos equates to what is meant by the adaptive approach as defined in this research, the deliberate-emergent approach. The view that organizations should take an adaptive approach is echoed by Eisenhardt and Brown [9]. This deliberate versus the emergent approach is applied to the four key elements proposed by ScottMorton MIT90's framework [2]: adaptive strategy; adaptive business processes; adaptive strategy organizational structures; and adaptive technology (information systems). This results in a prescription for an ASO as illustrated in figure 1, which is synthesized from Scott-Morton MIT90's framework [2] and types of strategies proposed by Mintzberg and Waters [10].

The idea of an adaptive organization has been defined as a hybrid concept in which both Deliberate and Emergent approaches are practiced [4]. The derivation of this definition comes primarily from the work of Scott Morton [2] who proposed that an organization can be viewed as a composition of interrelated factors. Following this, Scheer [8] suggested that organizations were able to follow either a stable yet inflexible (Deliberate) or a reactive and flexible (Emergent) approach. Mintzberg [11] stipulated that striking a balance between both approaches was optimal for adaptability, thus forming the definition above. The combination of Scott Morton's [2] and Mintzberg's [11] work therefore 
provides a foundation for the discussion of an adaptive organization. SOPI factors are inherent within the MIT90 framework in relation to adaptability and helps provide insight into the operations of an adaptive organization [4][7]. Different approaches (Emergent and Deliberate) to managing the SOPI components determine the level of adaptability. This approach follows Haeckel's [12], [13] conceptualization of an adaptive organization in which it is stated that a truly adaptive organization would maintain its operations in a specific manner. The manner alluded here can be argued to be in relation to the Emergent and Deliberate approaches discussed above.

\subsection{Sustainable View}

If we are to embrace and meet sustainability challenges we first need to understand the concept of sustainability as it applies to this research. Sustainability is about the ability and capacity to continue, to endure and to strategize for the present and the future. In economic terms, that means an organization takes the necessary measures for it to stay in business, or in other words, to be economically sustainable. In terms of the societal sustainability dimension, it means an organization should make decisions based on the knowledge of how those decisions will affect people and the repercussions on society in general. Organizations need to take the steps necessary to assure that its very existence will have a positive, rather than a negative effect on society and the cultures of people its decisions affect. In terms of the influence an organization may have on the environment, to be sustainable means to attempt to be a caretaker of the planet, to maintain the natural world and be responsible for not harming the environment to the extent that future generations will suffer from the damage or the change to the environment. These three dimensions are captured in figure 1. To be truly sustainable an organization needs to successfully balance the economic, environmental, and societal dimensions.

The definition of sustainability has many interpretations but is also often described in terms of 3 dimensions (People, Planet, and Profit), also referred to as the Triple Bottom Line [14]. Overall, sustainability is achieved through following the demands of having a minimum impact on materials with maximum benefit for society, as well as making a positive impact on communities and societies without a long-term negative impact on the world's ecological systems [15].

Furthermore, the concept of sustainability and sustainable development [16] is becoming a strategic imperative for many organizations [17]. Sustainable practices are a critical part of the organization's acceptable business norms [18] [19] and implemented through the organizational elements of strategy, structure, processes, individuals and roles, and technologies [20].

A sustainable perspective of an organization is a developing concept. The concept of Adaptive Organizations has been widely researched with a plethora of literature about AE from many different perspectives. However such literature has lacked discussion on Organizations that are both adaptable and sustainable. We explore this concept and define sustainability in terms of sustaining survival and productivity while also suggesting that sustainability be defined in terms of Economics, Culture, Environment, and Society. This is a step-up from the standard Triple Bottom Line criteria that authors have previously used to define sustainability and supports the proposition that sustainability is a multidimensional construct [21]. The four dimension model proposed can be likened to the SOPI factors discussed for the adaptive view as these factors must also be aligned in a manner which optimizes sustainability

\section{Systems View of an Adaptive Sustainable Organization}

There is little understanding of how sustainable systems and adaptive systems can be leveraged and interwoven with organization systems designed around strategy, business processes, organizational structures and information systems [22]. There is sparse literature on how to design and support such systems, along with their development. We propose a new model of an ASO that attempts to interweave the adaptive dimension (deliberate and emergent) and the sustainable dimension (economic, environmental, and societal) and organization dimension (strategy, process, organization and systems) in a seamless way (see figure 1).

The overarching objective of our research is to explore how "an organization can become both adaptive and sustainable by interweaving the deliberate and emergent in the context of strategy, business processes, organizational structures and information systems, along with systems that support the three main sustainability dimensions, namely the environmental, economical and societal concerns?"

The conceptualization of a systems view of an ASO requires an amalgamation of the perspectives discussed thus far. Each of the views discussed contributes to how an ASO can be regarded as a system. Firstly however, there must be consideration of what an ASO entails. The previous section explored adaptability and 
sustainability in their own right, however the focus must shift to how these factors can be considered in conjunction. Building on the model of an ASO it can be said that an ASO will take the SOPI factors and orientate them according to the sustainability dimensions discussed whilst also maintaining adaptive approach. This suggests that a factor such as Strategy will not only be following elements of emergent and deliberate approaches but also accommodating elements of sustainability. Here an ASO is defined as an organization which simultaneously displays adaptive and sustainable practices by applying both deliberate and emergent approaches to strategy, organizational structure, business processes, and information systems while also incorporating dimensions of sustainability into its practices. Defining an ASO in this way thus leads to the systems view as operations will only be viable if the organization operates systematically.

Firstly considering the hierarchical view, it has been established that the layers will operate in a systematic way by aligning to the desired goal and thus satisfy the adaptability component. An additional element here is that the layers will also incorporate the sustainability dimensions from top to bottom. The hierarchical view can be considered in conjunction with the functional view that proposes that an organization can be considered a system on the basis that the functional silos are interrelated. Considering these two views together suggests a system whereby there is horizontal and vertical integration throughout the organization and this is exactly what is expected of an ASO.

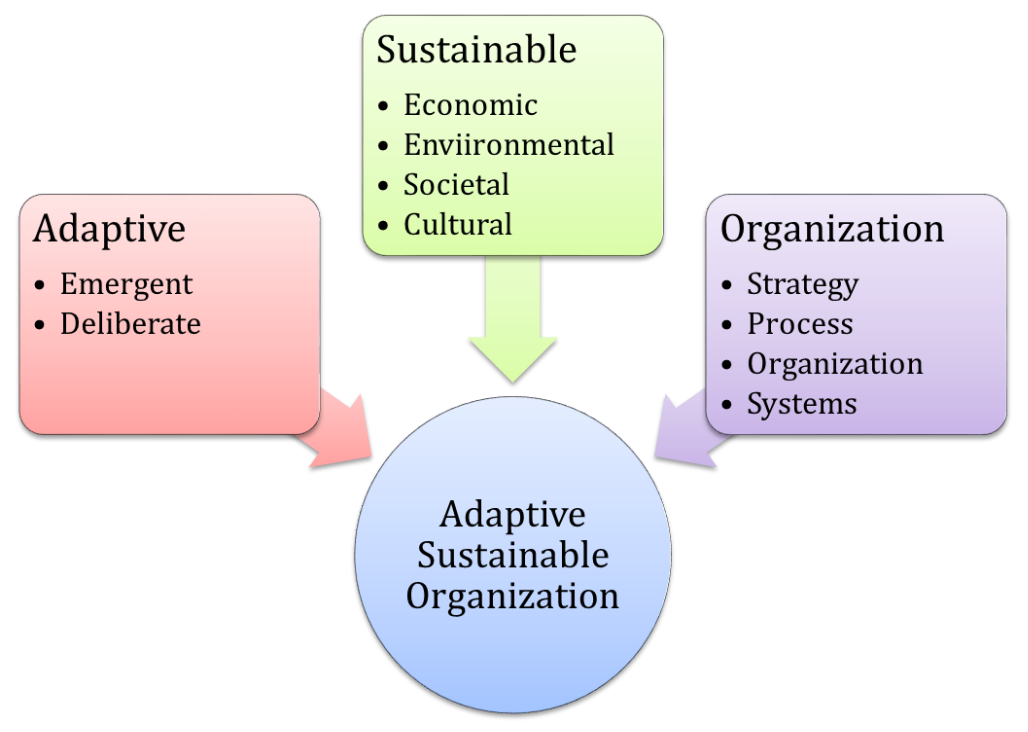

Figure 1. Adaptive Sustainable Organization

The concepts and perspectives considered allow for the development of an ASO architecture (figure 2) which demonstrates the systems view intended to be emphasized. The model builds on the architecture proposed in Peko et al. [4] particularly through the addition of features which reflect a systems view. As suggested by the key, there are three forms of flows to be considered under the systems view: Control flow, Information flow, and Learning flow. Control flow alludes to the manner in which an organization is controlled, which in this case is through a top down approach as well as through sustainable practices. Information flow is focused on how information is collected by an organization and here this is through bottom up approaches as well as from external sources. The final flow is the Learning flow which aims to continuously develop understandings of the organization through performance management and the sustainability elements with the aim of maintaining adaptability.

\section{Viewing a University as an Adaptive Sustainable Organization}

\subsection{Context}

The operations of an ASO is best illustrated by considering an entity currently oriented towards adaptability and sustainability. For the purposes of this paper, a university is used as the organization in question. In particular the focus is on a Research 
Driven University (RDU) which as the name suggests, is a university which focuses primarily on research. The key performance indicator for an RDU would take into consideration is its rankings (e.g. QS World University Rankings). This is highly influenced by the extent of research success which is measured by both the quality and quantity of the research output. This success in turn translates to revenue for the RDU through stakeholders and benefactors of the university who will be more inclined to provide grants and funding. This revenue is considered the primary source of income for an RDU. The secondary source of income for such a university would be the revenue derived through teaching activities, namely the fees paid by students in exchange for tuition. Given the research orientation, it is expected that employees would dedicate a greater proportion of their time to research-based activities rather than teaching-based activities. This gives rise to a key trade-off between the time allocated to teaching and research and this forms the basis for viewing the university as a system. The development of a model for an organization such as a university requires the application of systems dynamics. It is suggested by Maani and Cavana [23] systems thinking techniques are crucial in understanding complex systems and a university would fall under such a category given the number of potential dimensions.

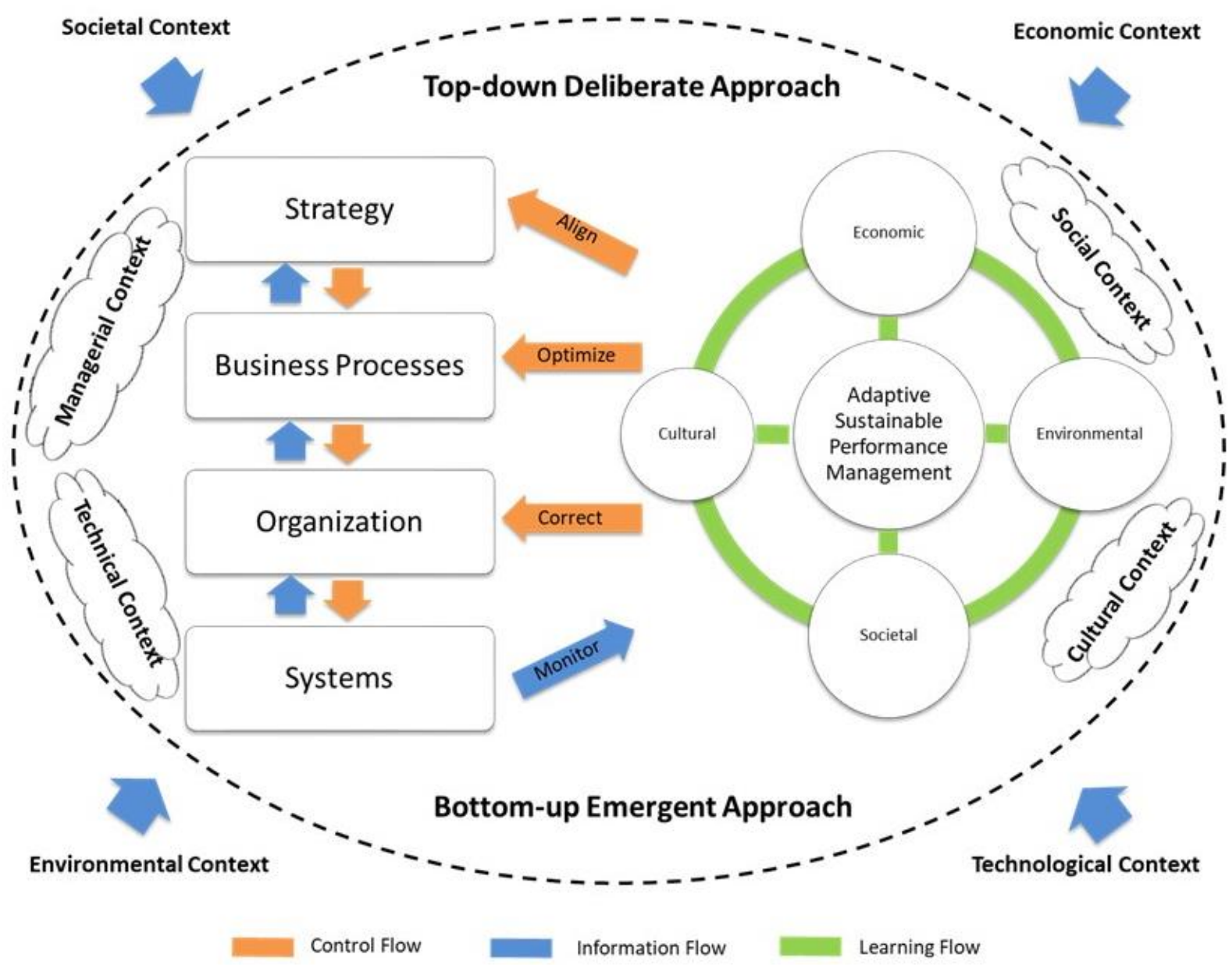

Figure 2. Systems view of an Adaptive Sustainable Organization

In modelling the dynamics of an adaptive and sustainable university, we apply standard system dynamics theory and model the whole system rather than isolate each component. A university can therefore be viewed as one system with the key activities of teaching and research being the major components. Modelling the whole system illustrates the potential adaptive and sustainable nature of a 
university as we can observe how the dynamics of teaching and research vary in order for the institution to maximize the economic element. The dynamics of the system also support discussions in relation to cultural and societal elements of the four dimension sustainability model.

However as with any model, there are inherent difficulties in illustrating all variables within one model and so secondary sub models have also been developed to highlight other key relationships within the system. These sub models provide insight into how factors such as quality and perception of teaching can also contribute to sustainability.

\subsection{University as a System}

The core model developed to illustrate the systems view of an RDU is presented in figure 3. The key component of the model is the Teaching Time module which is partially a decision made by a staff member but also determined by the departmental decision making personnel who allocate teaching loads to employees. Once this has been determined, the model begins to exhibit dynamic behavior through cause and effect. The Teaching Time directly influences the Teaching Quality which in turn influences the extent of Student Learning. Student Learning affects the quality and quantity of Research Students produced which then results in a change in the quality and quantity of Research Output. Research Output is also dependent on the Research Time allocated and the resulting output will determine the extent of Recognition received which is measured in terms of promotion and reputation.

The Recognition then influences the amount of Teaching Time an employee allocates. Student Learning also influences the amount of Positive Feedback that the teaching employee receives. According to the level of positive feedback, the Teaching Load will be altered which will then affect the Teaching Time. Furthermore the key trade-off between Research Time and Teaching Time is also reflected with Teaching Time changing in the opposite direction to the change in Research Time.

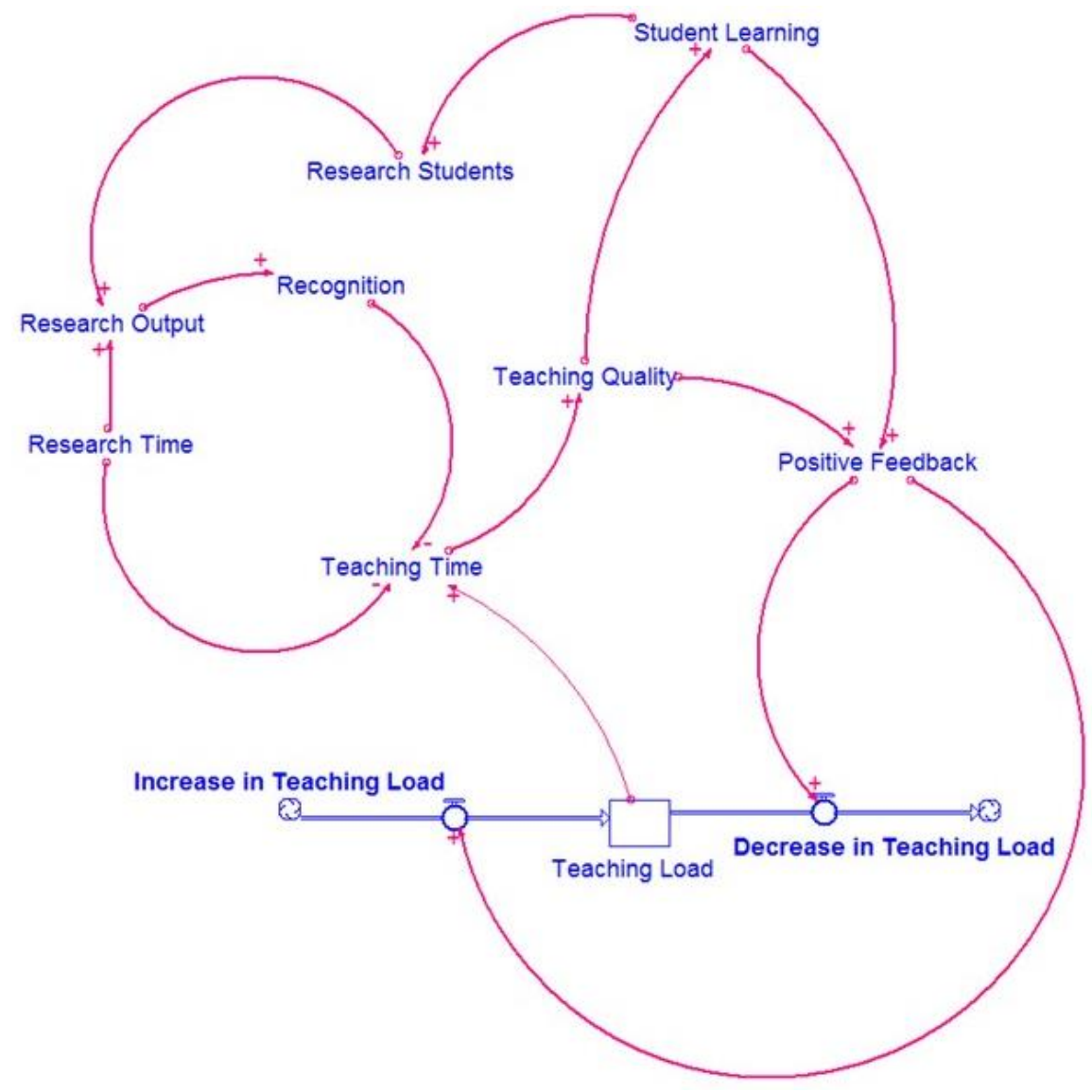

Figure 3. iThink causal loop diagram of a university 
The model illustrates that a university can behave in a dynamic manner by self-regulating and adapting according to the circumstances. The model can enter both a vicious or a virtuous cycle in adherence with the situation and this is discussed below.

\subsection{The Vicious AS-IS Descriptive Cycle}

The vicious cycle in figure 4 highlights the scenario in which Teaching Time decreases and the flow on effects result in a negative loop whereby Teaching Time continues to diminish. As seen on the model, the reduction in Teaching Time has an adverse effect on the Teaching Quality which reduces the level of Student Learning and the amount of Positive Feedback received. This leads to a decision to reduce the Teaching Load which reduces Teaching Time further.
Following the model through the other pathway, it is observed that reduced Student Learning diminishes Research Students which in turn reduces Research Output. Simultaneously Research Time increases (as Teaching Time decreases) which contributes to greater Research Output. Here it is observed that there is conflicting pathways with both an increase and a decrease feeding into Research Output. This arises due to the dynamic nature of the model which dictates an attempt to self-correct the vicious cycle. In this case the decrease in Research Output overpowers the increase (thus defeating the self-correcting mechanism). Consequently it is seen that Recognition is decreased and employees will be even more inclined to limit their Teaching Time to dedicate more time to Research in hopes of eventually acquiring greater Recognition. It is in this manner that the vicious cycle continues, lessening the Teaching Time even further.



Figure 4. iThink causal loop diagram of a university: vicious descriptive cycle 
As we can see, universities quite often take decisions driven by economic and funding requirements/constraints/goals which while seeming to improve the financial status in the short term often leads to loss in terms of contribution/value to society.

\subsection{The Virtuous TO-BE Normative Cycle}

The virtuous cycle in this model, depicted in figure 5 , focuses on the scenario whereby Teaching Time increases over time. The cycle is akin to the vicious cycle except with polar opposite changes for most modules. The cycle once again begins with Teaching Time but here an increase is shown and this has positive effects on Teaching Quality, Student Learning, and the Positive Feedback received. This in turn leads to the decision to increase Teaching Load thus increasing the Teaching Time for a particular employee. In terms of the other pathway, increased Student Learning also leads to positive changes in Research Students and Research Output.

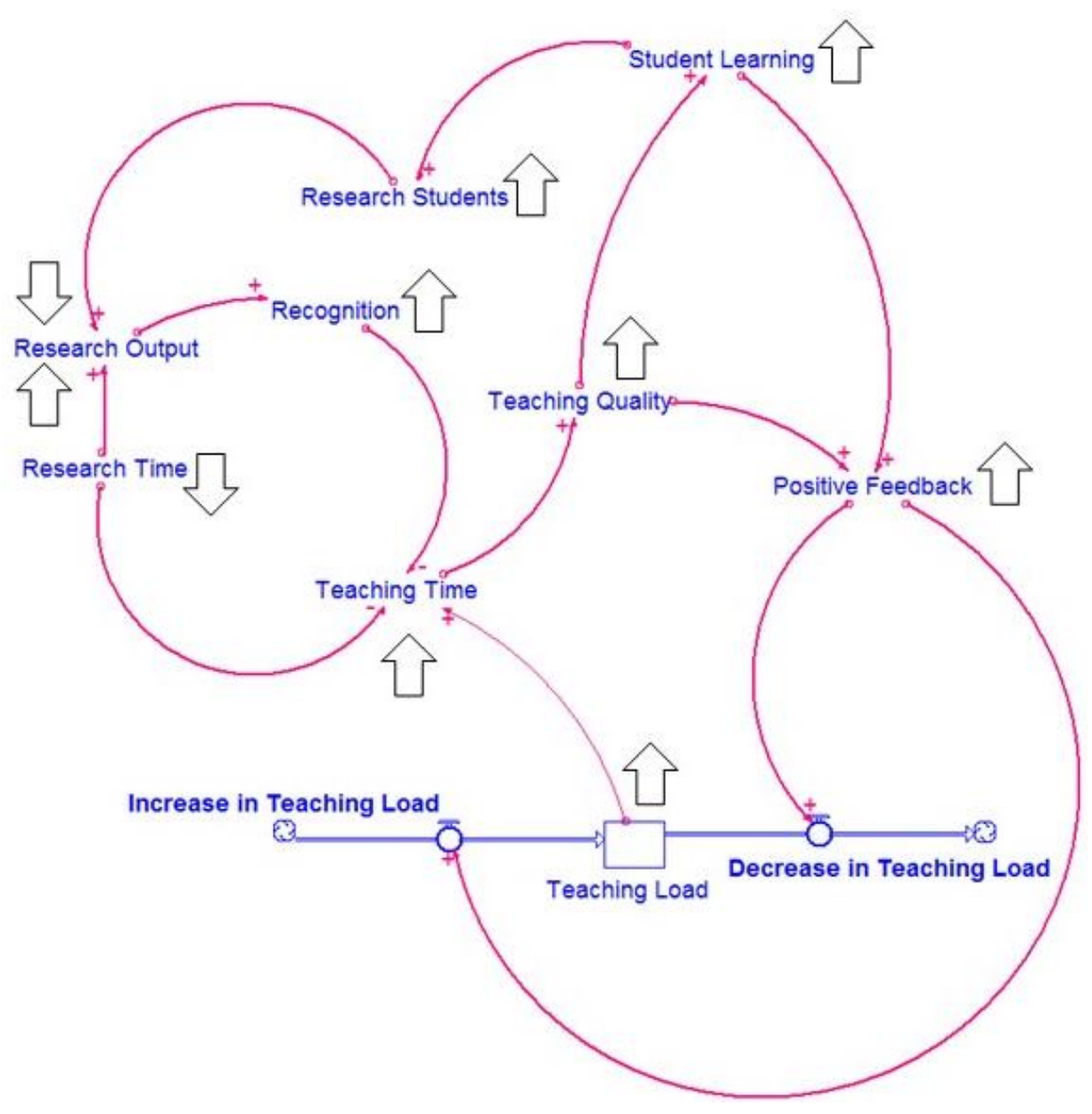

Figure 5. iThink causal loop diagram of a university: virtuous normative cycle

However as previously observed, once again there is a conflict with Research Time decreasing due to the increase in Teaching Time. This time the conflict is overpowered by the increase in Research Output and subsequently there is an increase in Recognition. The increase in Recognition however encourages employees to make the decision to dedicate more time to Research at the expense of Teaching. However since they have shown themselves to be dedicated teachers, the university system burdens them with more teaching.

\subsection{Discussion}

The various outcomes arising from the vicious and virtuous cycles illustrate the application of the ASO 
framework. In particular, the Control, Information, and Learning flows can be observed. Control is seen through the key decision on teaching loads. The decision from top management is likely to be driven by economic motives and the nature of this decision determines whether the vicious or virtuous cycle manifests. The Information flow, although not directly visible, is inherently present within the cycles. The feedback flows, in particular, represent how information from the bottom level, i.e. the students influence decisions at the top. The Learning flow is represented by the understanding developed over time which should result in a movement away from the ASIS model and towards the virtuous TO-BE cycle. With these three flows, we can understand how an RDU can align, optimize, correct, and monitor to be adaptive and sustainable

\section{Conclusion and Future Research}

The research conducted in the area of adaptive and sustainable Organizations has experienced growth over the years, particularly with the increased incorporation of sustainability dimensions. To build upon and contribute to this research, a new perspective is applied to the adaptability and sustainability characteristics. The literature considered focuses on the different views of an organization and this supports the defining of an ASO. From there a systems view was applied to an ASO to understand the organization in a different light and in doing so, an architecture was developed to support the view. To further elaborate on the view, universities were exemplified as an organization and modelled to provide insight into the systems view put forward. We explored vicious and virtuous cycles of adaptive behavior within a university context and the potential to adapt in sustainable ways. Looking ahead, there still remains to be greater focus on the sustainability dimensions apart from the economic one. Although briefly discussed, the dimensions are definite viable options for discussions in greater depth.

\section{References}

[1] Anthony, R. N., Planning and Control Systems: A Framework for Analysis, Cambridge MA, Harvard University Press, 1965.

[2] Scott Morton, M. S., The Corporation of the 1990s, Oxford University Press, 1991.

[3] Von Bertalanffy, L., General Systems Theory Foundations, Development, Applications, George Braziller, New York, 1968.
[4] G. Peko, C. Dong, and D. Sundaram, "Adaptive Sustainable Enterprises", Mobile Networks and Applications, (19:5), 2014, pp. 608-617.

[5] Loucopoulos, P., and V. Kavakli, Organization knowledge management and conceptual modelling. In Conceptual Modeling, Springer, Berlin, Heidelberg, 1999, pp. 123-143.

[6] A.W. Scheer, ARIS - Business Process Frameworks, 3rd ed., Springer, Berlin, Heidelberg, 2000.

[7] Peko G., Adaptive Organizations Interweaving the Deliberate and Emergent - Concepts and Models, unpublished doctoral dissertation, University of Auckland, Auckland, New Zealand, 2017.

[8] A.W. Scheer, "Jazz Improvisation and Management", IDS Scheer AG, 2007, http://whitepaper.talentum.com/whitepaper/view.do?id= $\underline{21050}$ accessed 12 November 2017.

[9] K.M., Eisenhardt, and S.L. Brown, "Competing on the edge: Strategy as structured chaos", Long Range Planning, 31(5), 1998, pp. 786.

[10] H. Mintzberg, and J.A. Waters, "Of strategies, deliberate and emergent", Strategic management journal, 1985, pp. 257-272.

[11] H. Mintzberg, "The fall and rise of strategic planning", Harvard Business Review, 1994, pp. 107.

[12] Haeckel, S., Adaptive Enterprise, 2nd ed., Harvard Business School Press, Boston, Mass., 2007, pp. 3-4.

[13] Haeckel, S., Adaptive Enterprise: The Work Book, Createspace Independent Publishing Platform, United States, 2016.

[14] J. Elkington, "Partnerships from cannibals with forks: The triple bottom line of 21st-century business", Environmental Quality Management, (8), 1998, pp. 3751.

[15] Ferguson, G., A. Dakers, and I. Gunn, Sustainable Waste Management: A Hand Book for Smaller Communities, (Vol. 477), New Zealand: Ministry for the Environment, 2004.

[16] C.H. Daub, and R. Ergenzinger, "Enabling sustainable management through a new multi-disciplinary concept of customer satisfaction", European Journal of Marketing, 39(9/10), 2005, pp. 998-1012.

[17] R.J. Baumgartner, "Organizational culture and leadership: Preconditions for the development of a sustainable corporation", Sustainable Development, 17(2), 2009, pp. 102-113.

[18] E. Collins, S. Lawrence, K. Pavlovich, and C. Ryan, "Business networks and the uptake of sustainability 
practices: the case of New Zealand", Journal of Cleaner Production, 15, 2007, pp. 729-740.

[19] D.A. Lubin, and D.C. Esty, "The sustainability imperative", Harvard Business Review, 88, 2010, pp. 42-50.

[20] Epstein M.J., and H.B. Leonard, Making sustainability work: Best practices in managing and measuring corporate social, environmental and economic impacts, Greenleaf, Sheffield, 2008.

[21] M.D. Ahmed, and D. Sundaram, "Sustainability modelling and reporting: From roadmap to implementation, Decision Support Systems", 2012, 53(3), pp. 611-624.

[22] G. Weichhart, A. Molina, D. Chen, L.E. Whitman, and F. Vernadat, "Challenges and current developments for Sensing, Smart and Sustainable Enterprise Systems", Computers in Industry, 2016, pp. 79.

[23] Maani, K., and R. Cavana, Systems thinking and modelling, 2nd ed., Pearson Education, Auckland, New Zealand, 2009, pp. 7-49. 